\title{
Critical behavior of the domain wall collapse and oscillon
}

Taishi lkeda*†

Nagoya University

E-mail: ikedadqravity.phys.nagova-u.ac.ip

In this proceedings, we consider the gravitational collapse of a spherically symmetric domain wall, and its critical collapse. By numerical simulation, we show that the type II critical collapse associated with discrete self-similar solution appears, and the index and the period of the mass scaling agree with the massless scalar case.

The 3rd International Symposium on "Quest for the Origin of Particles and the Universe" 5-7 January 2017

Nagoya University, Japan

* Speaker.

${ }^{\dagger}$ A footnote may follow. 


\section{Introduction}

General relativity is the most successful theory of gravity. Although this theory has been studied for about 100 years, because the field equation is nonlinear equation, its behavior is nontrivial. So, numerical relativity is a powerful method to understand the nonlinear phenomena. About 20 years ago, Choptuik studied a gravitational collapse of a spherically symmetric massless scalar system by using numerical relativity, and discovered a critical collapse, which is a universal behavior of gravitational system.[四][四]

In this study[], we focus on the spherically symmetric domain wall collapse in the following system:

$$
S=\int d^{4} x \sqrt{-g}\left\{\frac{R}{16 \pi}-\frac{1}{2} \nabla_{\mu} \Phi \nabla^{\mu} \Phi-V(\Phi)\right\},
$$

and discuss the critical behavior of a domain wall collapse. The critical collapse of this system is discussed in [Q], and type II critical collapse appears. Nevertheless, since this system has a typical

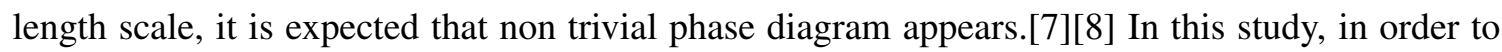
investigate the non trivial phase diagram of this system, we study the critical collapse of this system in different parameter space.

\section{Method}

In order to analyze the time evolution of the gravitational collapse of the spherically symmetric domain wall, we developed the numerical code. In this section, we explain our formulation and construction of the initial data.

\section{1 formulation}

We take a spherical metric ansatz as follows:

$$
d s^{2}=-\alpha^{2}(t, r) d t^{2}+\psi^{4}(t, r)\left\{\gamma(t, r)^{-2}(d r+r \beta(t, r) d t)^{2}+\gamma(t, r) r^{2} d \Omega^{2}\right\},
$$

where $d \Omega^{2}$ is the solid angle element, and $\psi, \alpha, \beta$ and $\gamma$ are independent functions of $t$ and $r$. Because the extrinsic curvature $K_{i j}$ of each time slice has two independent components, we can decompose $K_{i j}$ into $K$ and A, which are defined as follows:

$$
K \equiv \gamma^{i j} K_{i j}, A \equiv\left(K_{\theta \theta}-\frac{1}{3} K \gamma_{\theta \theta}\right) /\left(\psi^{4} r^{2}\right),
$$

where $\gamma_{i j}=\psi^{4} \operatorname{diag}\left(\gamma^{-2}, \gamma r^{2}, \gamma r^{2} \sin ^{2} \theta\right)$ is a special metric. Substituting the metric form (R.T) into the Einstein equations we get the following time evolution equations:

$$
\begin{aligned}
& \left(\partial_{t}-r \beta \partial_{r}\right) \psi=\frac{1}{6} \psi\left(3 \beta+r \beta^{\prime}-\alpha K\right), \\
& \left(\partial_{t}-r \beta \partial_{r}\right) K=\alpha\left\{\frac{1}{3} K^{2}+6 \frac{A^{2}}{\gamma^{2}}+8 \pi \Pi^{2}-8 \pi V(\Phi)\right\}-\psi^{-4} \gamma^{2}\left\{\Delta \alpha+2 \alpha^{\prime}\left(\frac{\psi^{\prime}}{\psi}+\frac{\gamma^{\prime}}{\gamma}\right)\right\} \\
& \left(\partial_{t}-r \beta \partial_{r}\right) \gamma=-2 \alpha A-\frac{2}{3} r \gamma \beta^{\prime}
\end{aligned}
$$




$$
\begin{aligned}
\left(\partial_{t}-r \beta \partial_{r}\right) A= & \alpha K A-2 \alpha \frac{A^{2}}{\gamma}-\frac{2}{3} r A \beta^{\prime}+\psi^{-4}\left\{-\frac{1}{6} \gamma^{3}\left(\Delta \alpha-3 \alpha^{\prime \prime}\right)-\frac{1}{3} \alpha \gamma^{3}\left(\frac{\Delta \psi}{\psi}-3 \frac{\psi^{\prime \prime}}{\psi}\right)\right. \\
& -\frac{1}{6} \alpha(1+\gamma) \Delta \gamma+\frac{1}{6} \alpha\left(1+\gamma+\gamma^{2}\right) \gamma^{\prime \prime}-\frac{1}{3} \alpha\left(1+\gamma+\gamma^{2}\right)\left(-\frac{\gamma-1}{r^{2}}+\frac{\gamma^{\prime}}{r}\right)+\frac{1}{6} \alpha^{\prime} \gamma^{2} \gamma^{\prime} \\
& \left.-\frac{4}{3} \alpha^{\prime} \gamma^{3} \frac{\psi^{\prime}}{\psi}+\frac{1}{3} \alpha \gamma^{2} \gamma^{\prime} \frac{\psi^{\prime}}{\psi}-2 \alpha \gamma^{3} \frac{\psi^{\prime 2}}{\psi^{2}}+\frac{8}{3} \pi \alpha \gamma^{3} \Phi^{\prime 2}\right\} \\
\left(\partial_{t}-r \beta \partial_{r}\right) \Phi= & -\alpha \Pi, \\
\left(\partial_{t}-r \beta \partial_{r}\right) \Pi= & \alpha \Pi K-\psi^{-4} \alpha \gamma^{2}\left\{\Delta \Phi+2 \Phi^{\prime}\left(\frac{\gamma^{\prime}}{\gamma}+\frac{\psi^{\prime}}{\psi}+\frac{\alpha^{\prime}}{2 \alpha}\right)\right\}+\alpha V^{\prime}(\Phi),
\end{aligned}
$$

where $\Pi$ is the conjugate momentum of $\Phi$. The constraint equation is as follows:

$$
\begin{aligned}
\frac{\Delta \psi}{\psi}+\frac{1}{8}\left(5 \frac{\Delta \gamma}{\gamma}-3 \frac{\gamma^{\prime \prime}}{\gamma}\right)+ & \pi \Phi^{\prime 2}+2 \pi \gamma^{-2} \psi^{4} V(\Phi)+\frac{\left(\gamma^{2}+\gamma+1\right)(\gamma-1)}{4 \gamma^{3} r^{2}} \\
+\frac{\gamma^{\prime}}{\gamma}\left(2 \frac{\psi^{\prime}}{\psi}+\frac{3}{16} \frac{\gamma^{\prime}}{\gamma}\right)+\frac{\psi^{4}}{\gamma^{2}}\left(\frac{3 A^{2}}{4 \gamma^{2}}+\pi \Pi^{2}-\frac{1}{12} K^{2}\right) & =0, \\
A^{\prime}+\frac{\gamma}{3} K^{\prime}+4 \pi \gamma \Pi \Phi^{\prime}+\frac{3 A}{r}+\frac{A \gamma^{\prime}}{2 \gamma}+6 A \frac{\psi^{\prime}}{\psi} & =0 .
\end{aligned}
$$

We developed the numerical code based on the above formulation.

\subsection{Initial data}

The initial profile of this study is the momentary static domain wall. From the momentary static condition, we assume that $K, A$ and $\Pi$ vanish on the initial time slice. In order to express the domain wall we assume that the profile of the scalar filed is as follows:

$$
\Phi(r, t=0)=\sigma \tanh \left(\frac{r-r_{0}}{l}\right)+\sigma\left\{-1-\tanh \left(\frac{r-r_{0}}{l}\right)\right\} \exp \left\{-\left(\frac{r}{l}\right)^{4}\right\}
$$

where first term corresponds to the domain wall profile, second term corresponds to the regularization term,,$l$ is a width of the domain wall which is given by $l=\frac{2}{\sigma} \sqrt{\frac{3}{\lambda}}$, and $r_{0}$ is a initial radius of the domain wall In this study, we investigate the relation between the initial radius of the domain wall $r_{0}$ and the mass of the black hole $M_{\mathrm{BH}}$.

\section{Result}

We calculate the time evolution, and get the relation between the initial radius of a domain wall and mass of a black hole (see Fig.W). As is shown in Fig.W, the relation between $r_{0}$ and $M_{\mathrm{BH}}$ obeys the scaling low with periodic function: $\ln M_{\mathrm{BH}}=v \ln \left|p-p_{*}\right|+c+f\left(\ln \left|p-p_{*}\right|\right)$. [䧃] The indexes $v$ of the mass scaling are 0.370 .38 , and it agrees with the massless scalar case. Furthermore, we could check the period of the fine structure also agree with the massless scalar case.[四]

\section{Summary and discusion}

In this study, we investigate the critical behavior of a spherically symmetric domain wall collapse. By numerical simulation, we show that the type II critical behaviors appears, and the index of the mass scaling and the period of its fine structure agree with the massless scalar case. 

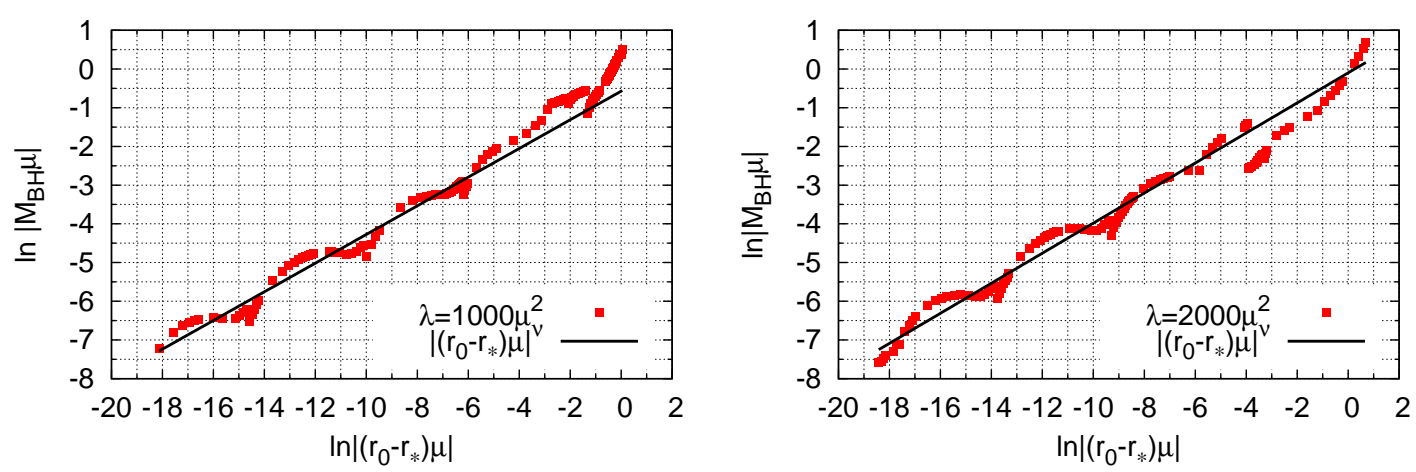

Figure 1: The left panel shows the mass scaling for the $\lambda=1000 \mu^{2}$ case, and the right panel shows the mass scaling for the $\lambda=2000 \mu^{2}$ case.

Since this system has a typical length scale, it is expected that type I critical behavior also appears. (see [[]][[]]) In the case of the massive scalar field, the soliton star which is long life time localized solution plays an important role in the non-trivial phase diagram. In our system, although no body know such a longevity solution, there is such solution in the scalar filed with double well potential in Minkwski spacetime, which is called as Oscillon. Therefore, if we could construct the Oscillon with gravity, it is expected that it plays the important role in the nontrivial phase diagram of the domain wall collapse. The construction of the Osciilon with gravity is future work of this study.

\section{References}

[1] M. W. Choptuik, Phys. Rev. Lett. 70, 9 (1993).

[2] T. Ikeda and C. M. Yoo, Phys. Rev. D 94, no. 12, 124032 (2016) doi:10.1103/PhysRevD.94.124032 [arXiv: 1610.07280 [gr-qc]].

[3] C. Gundlach and J. M. Martin-Garcia, Living Rev. Rel. 10, 5 (2007) [arXiv:0711.4620 [gr-qc]].

[4] S. Hod and T. Piran, Phys. Rev. D 55, 440 (1997) [gr-qc/9606087].

[5] D. Garfinkle and G. C. Duncan, Phys. Rev. D 58, 064024 (1998) [gr-qc/9802061].

[6] P. R. Brady, C. M. Chambers and S. M. C. V. Goncalves, Phys. Rev. D 56, R6057 (1997) [gr-qc/9709014].

[7] H. Okawa, V. Cardoso and P. Pani, Phys. Rev. D 89, no. 4, 041502 (2014) [arXiv:1311.1235 [gr-qc]].

[8] K. Clough and E. A. Lim, arXiv:1602.02568 [gr-qc]. 